\title{
OMITTED VALUES IN DOMAINS OF NORMALITY
}

\author{
WALTER BERGWEILER AND STEFFEN ROHDE
}

(Communicated by Albert Baernstein II)

\begin{abstract}
It is proved that if $U$ and $V$ are connected components of the Fatou set of an entire function $f$ and if $f(U) \subset V$, then $V \backslash f(U)$ contains at most one point.
\end{abstract}

Let $f$ be an entire (or rational) function. The Fatou set $F$ of $f$ is the subset of the plane (or sphere) where the iterates of $f$ form a normal family. It is easy to see that if $U$ is a connected component of $F$, then $f(U)$ is contained in some connected component $V$ of $F$. For rational functions, we have $f(U)=V($ see $[2, \S 5.4])$. For transcendental entire functions, it is possible that $f(U) \neq V$. A simple example is given by $f(z)=\lambda e^{z}, 0<\lambda<e^{-1}$, where $F$ is connected and $0 \in F \backslash f(F)$.

Theorem. Let $f$ be an entire transcendental function and let $U$ and $V$ be connected components of $F$ satisfying $f(U) \subset V$. Then $V \backslash f(U)$ contains at most one point.

To prove the theorem, let $f, U$, and $V$ be as required and suppose that $V \backslash f(U)$ is not empty. It is easy to see that this implies that there exists a curve $\gamma$ tending to $\infty$ in $U$ such that $f(z)$ tends to a value in $V \backslash f(U)$ as $z \rightarrow \infty$ in $\gamma$. In particular, $f(z)$ is bounded on some curve tending to $\infty$. A result of Baker $[1, \S 3]$ now implies that $U$ and $V$ are simply-connected. Hence there exist conformal maps $\varphi$ and $\psi$ from the unit disk $D$ onto $U$ and $V$. We define $g=\psi^{-1} \circ f \circ \varphi$ so that $g(D) \subset D$. Clearly, it suffices to prove that $D \backslash g(D)$ contains at most one point.

By a result of Beurling (see [4, Theorems 11.5 and 11.9]) there exists a set $A \subset[0,2 \pi]$ of capacity zero with the property that if $\theta \notin A$, then there exists $a_{\theta} \in \partial U \backslash\{\infty\}$ such that $\varphi\left(r e^{i \theta}\right) \rightarrow a_{\theta}$ as $r \rightarrow 1$. It follows that $f\left(\varphi\left(r e^{i \theta}\right)\right) \rightarrow$ $f\left(a_{\theta}\right) \in \partial V \backslash\{\infty\}$ and hence that $\left|g\left(r e^{i \theta}\right)\right| \rightarrow 1$ as $r \rightarrow 1$, provided $\theta \notin A$. A result of Lohwater (see [3, Theorem 5.14]) now implies that $D \backslash g(D)$ contains at most one point. This completes the proof of the theorem.

Received by the editors October 19, 1993.

1991 Mathematics Subject Classification. Primary 30D05, 58F08.

Key words and phrases. Iteration, entire function, set of normality, Fatou set, Julia set. 


\section{ACKNOWLEDGMENT}

We would like to thank Professor I. N. Baker for bringing to our attention that this result has been proved independently by M. Herring. We are also grateful to the Mathematisches Forschungsinstitut Oberwolfach for hospitality and to the Studienstiftung des Deutschen Volkes for financial support.

\section{REFERENCES}

1. I. N. Baker, Wandering domains in the iteration of entire functions, Proc. London Math. Soc. (3) 49 (1984), 563-576.

2. A. F. Beardon, Iteration of rational functions, Springer, New York, Berlin, and Heidelberg, 1991.

3. E. F. Collingwood and A. J. Lohwater, The theory of cluster sets, Cambridge Univ. Press, London and New York, 1966.

4. Chr. Pommerenke, Univalent functions, Vandenhoeck \& Ruprecht, Göttingen, 1975.

Lehrstuhl II für Mathematik, RWTH Aachen, D-52056 Aachen, Germany

Current address: Technische Universität Berlin, Fachbereich Mathematik, Sekr. MA 8-2, Strasse des 17. Yuni 136, D-10623 Berlin, Germany

Department of Mathematics, University of Michigan, ANn ARbor, Michigan 48109

Current address: Technische Universität Berlin, Fachbereich Mathematik, Sekr. MA 8-2, Strasse des 17. Yuni 136, D-10623 Berlin, Germany

E-mail address, S. Rohde: rohde@math.tu-berlin.de 